\title{
Cepabactin from Pseudomonas cepacia, a New Type of Siderophore
}

\author{
By JEAN-MARIE MEYER*, DANY HOHNADEL AND FÉLIX HALLÉ \\ Laboratoire de Microbiologie, Institut de Biologie Moléculaire et Cellulaire du Centre National \\ de la Recherche Scientifique, 4 rue Blaise Pascal, F-67070 Strasbourg Cedex, France
}

(Received 12 December 1988; accepted 15 February 1989)

\begin{abstract}
In iron-deficient conditions of growth Pseudomonas cepacia ATCC 25416 excreted both pyochelin and a low-molecular-mass compound which strongly chelated iron(III), and facilitated iron translocation as demonstrated by growth and uptake experiments. The name cepabactin is proposed for this new siderophore. Comparisons of UV-visible spectra and chromatographic behaviour, together with ${ }^{1} \mathrm{H}-\mathrm{NMR}$ spectra, led to the conclusion that cepabactin is 1-hydroxy-5-methoxy-6-methyl-2(1H)-pyridinone, a compound which can be considered as a cyclic hydroxamate, but also as a heterocyclic analogue of catechol. This pyridinone has already been described by other workers as an antibiotic produced by Pseudomonas alcaligenes, and by a soil isolate closely related to Pseudomonas cepacia. Thus, cepabactin appears to act as a siderophore for more than one species of non-fluorescent pseudomonad.
\end{abstract}

\section{INTRODUCTION}

Pseudomonas cepacia, first described as a phytopathogenic bacterium (Burkholder, 1950), has been reported since the last decade as a potential human pathogen isolated from a wide variety of clinical sources (Holmes, 1986). It belongs to the Pseudomonas pseudomallei RNA homology group within the DNA homology group II (Palleroni et al., 1973) and is remarkable by virtue of its ability to use over 100 different organic compounds as carbon and energy source (Stanier et al., 1966).

In previous studies of the iron metabolism of pseudomonads we found that pyoverdine, the yellow-green water-soluble pigment characteristic of Pseudomonas fluorescens, acts as a siderophore for this bacterium (Meyer \& Abdallah, 1978; Meyer \& Hornsperger, 1978). The iron-related properties of pyoverdine (called pseudobactin for plant-related isolates; Teintze et al., 1981) were common to almost all species belonging to the so-called fluorescent pseudomonads group (Leong, 1986; Meyer et al., 1987; Cody \& Gross, 1987; de Weger et al., 1988; Hohnadel \& Meyer, 1988). The siderophore system(s) of non-fluorescent pseudomonads, which are unable to synthesize pyoverdine, have been investigated for only a few species. Pseudomonas stutzeri under iron deficiency produced a colourless compound, able to strongly chelate iron(III), and chemically identified as desferri-ferrioxamine E (Meyer \& Abdallah, 1980). This compound is a cyclic trimer of succinic acid coupled via an amide bond to the hydroxyamino group of 1-amino-5-hydroxyaminopentane, thus resulting in the formation of a hydroxamate. It was first described as a secondary metabolite (sideramine) produced by Nocardia and several other actinomycetes, and called nocardamine (Stoll et al., 1951; Bickel et al., 1960; Keller-Schierlein \& Prelog, 1961 ; Müller \& Zähner, 1968). Although direct evidence of the involvement of nocardamine in iron transport is scant, its role as a siderophore was strongly suggested by its ability to enhance the growth of Arthrobacter JG-9 (Burnham \& Neilands, 1961) and other micro-organisms (Zähner et al., 1962) and by the fact that it reversed inhibition by

Abbreviation: EDDHA, ethylenediamine-di-(o-hydroxyphenylacetate). 
ferrimycin antibiotics (Prelog, 1963). Nocardamine stimulates the growth of $P$. stutzeri and reverses the growth inhibition caused by the synthetic iron chelator ethylenediamine-di( $o$ hydroxyphenylacetate) (EDDHA) (J.-M. Meyer, unpublished results). Uptake studies have demonstrated the participation of nocardamine in iron translocation by Streptomyces pilosus (Müller \& Raymond, 1984).

$P$. cepacia and clinical isolates belonging to this species have recently been reported to produce pyochelin (Bukovits et al., 1982; Sokol, 1986), a compound, chemically unrelated to pyoverdine, previously described in the fluorescent pseudomonad $P$. aeruginosa (Liu \& Shokrani, 1978; Cox \& Graham, 1979). A $14 \mathrm{kDa}$ ferripyochelin-binding protein, present in increased amounts in the outer membrane of iron-starved P. aeruginosa and P. cepacia cells, has also been described (Sokol $\&$ Woods, 1983; Sokol, 1984). Pyochelin has been shown to be a potent siderophore for clinical isolates of P. cepacia; and a correlation was found between its production and morbidity and mortality in infected cystic fibrosis patients, suggesting a role in pathogenicity (Sokol, 1986). However, about half of the clinical isolates investigated by Sokol (1986) were pyochelin negative. How these strains satisfy their iron requirement in an environment where iron is strongly chelated by host transferrin and lactoferrin remains unexplained. Moreover, pyochelin appears to be inefficient when competing for iron with the serum proteins. A $P$. aeruginosa mutant affected in the biosynthesis of pyoverdine $\left(\mathrm{Pvd}^{-}\right)$, but still able to produce pyochelin $\left(\mathrm{Pch}^{+}\right)$, had no observable advantage over a $\mathrm{Pvd}^{-} \mathrm{Pch}^{-}$mutant and had severely retarded growth compared to the wild strain in media supplemented with transferrin or human serum (Ankenbauer et al., 1985). Thus, even for the pyochelin-producing clinical isolates of $P$. cepacia, the existence of at least one supplementary iron-transport system would appear to be necessary for human pathogenicity.

\section{METHODS}

Strains and growth conditions. Pseudomonas cepacia ATCC 25416 was used throughout this work. Growth conditions were as described for Pseudomonas fluorescens by Meyer \& Abdallah (1978), using a synthetic medium with succinate as the carbon and energy source (succinate medium). One-litre Erlenmeyer flasks containing $500 \mathrm{ml}$ medium were used for large-scale preparation; growth experiments were done in $180 \times 18 \mathrm{~mm}$ capped glass tubes containing $7.5 \mathrm{ml}$ medium. Iron supplementation, when indicated, was performed by adding sterile $\mathrm{FeCl}_{3}$ solution ( $1 \mathrm{mg}$ iron $\mathrm{ml}^{-1}$ ) to the presterilized succinate medium. Media were inoculated with exponential-phase cells grown in the same medium, to give initial concentrations of $1 \%$ in volume for the $500 \mathrm{ml}$ cultures or $10^{6}$ to $10^{7}$ washed cells per $7.5 \mathrm{ml}$ for tubes. Unless otherwise indicated, incubation was at $25^{\circ} \mathrm{C}$ on a rotary shaker at 200 r.p.m. For comparison purposes the pyochelin-producer strain $P$. aeruginosa ATCC 15692 (strain PAO1) was grown under identical conditions

Growth and siderophore measurements. Growth in tube cultures was measured directly by using a Lumetron colorimeter (Photovolt Co.) with a $650 \mathrm{~nm}$ filter. Siderophore production was measured on $5 \mathrm{ml}$ culture samples. The sample was centrifuged for $10 \mathrm{~min}$ at $24000 \mathrm{~g}\left(4^{\circ} \mathrm{C}\right)$, and $10 \mu 12 \mathrm{M}-\mathrm{FeCl}_{3}$ was added to the supernatant and mixed vigorously. The supernatant was again centrifuged (same conditions) 30 min later, and the absorbance read on a Pye-Unicam SP8-400 (Philips) spectrophotometer. Depending on the pH of the culture at the time of harvest, the $\lambda_{\max }$ for the iron-siderophore complex in the visible region was between 440 and $460 \mathrm{~nm}$. Since only few differences in absorbance occurred within this range, the production of siderophore was quantified according to $A_{450}$.

${ }^{59} \mathrm{Fe}$ incorporation. Cells from $50 \mathrm{ml}$ of an exponential culture were harvested by centrifugation $(24000 \mathrm{~g}$, $10 \mathrm{~min}, 4^{\circ} \mathrm{C}$ ), washed twice with distilled water and resuspended at $\mathrm{OD}_{600} 0 \cdot 3$ in nitrogen-free succinate medium (incubation medium). Then $9 \mathrm{ml}$ of this suspension in a $250 \mathrm{ml}$ Erlenmeyer flask was incubated at $25^{\circ} \mathrm{C}$ in a shaking (100 r.p.m.) water-bath for $10 \mathrm{~min}$ before supplementation with $1 \mathrm{ml}$ of a labelled iron mixture (zero time) containing ${ }^{59} \mathrm{Fe}\left(5 \mu \mathrm{l}\right.$ of the commercial solution: chloride form, specific activity $20 \mathrm{mCi} \mathrm{mg}^{-1}, 740 \mathrm{MBq} \mathrm{mg}^{-1}$; Commissariat à l'Energie Atomique, Gif-sur-Yvette, France); $50 \mu \mathrm{l}$ of a $1 \mathrm{~mm}$ solution of the pure ligand dissolved in methanol and then diluted $1: 10$ with distilled water; and $945 \mu 1$ incubation medium, added 10 min later. Low blank values were obtained in control experiments without bacteria.

Samples $(1 \mathrm{ml})$ of the bacterial suspension supplemented with the labelled solution were withdrawn at intervals during the $30 \mathrm{~min}$ incubation, and rapidly filtered on to cellulose nitrate membrane filters (Whatman, $0 \cdot 45 \mu \mathrm{m}$ porosity). The filters were washed with $10 \times 2 \mathrm{ml}$ fresh incubation medium to remove non-incorporated labelled iron. When specified, KCN or 2,4-dinitrophenol (DNP) was added to the cell suspension 10 min before the addition of the iron source. In some experiments, cepabactin was replaced by EDDHA or various pyoverdines 
( $50 \mu \mathrm{M}$ final concentration). It was verified that under the conditions used, all these compounds completely solubilized the labelled iron.

Siderophore purification. The procedure used for cepabactin purification was essentially based on the method described by Barker et al. (1979) for the purification of antibiotic G1549, the trivial name given by the authors to 1-hydroxy-5-methoxy-6-methyl-2(1H)-pyridinone, which they isolated from Pseudomonas alcaligenes. Based on chloroform or ethyl acetate treatment of the acidified growth medium, this procedure is very similar to that used by Itoh et al. (1979) for the extraction of antibiotic BN-227 (the same pyridinone derivative as G1549) from Pseudomonas BN-227, or by Liu \& Shokrany (1978), Cox \& Graham (1979) or Sokol (1986) for the extraction of pyochelin from $P$. aeruginosa strains or clinical isolates of $P$. cepacia.

$P$. cepacia ATCC 25416 was grown in succinate medium (4 litres) supplemented with $2 \mu \mathrm{M}$-iron in order to maximize production of cepabactin (see Fig. 2). Cultures were centrifuged when cells reached the stationary phase $\left(40 \mathrm{~h}\right.$ of growth at $25^{\circ} \mathrm{C}$ ). The supernatant, acidified to $\mathrm{pH} 3.0$ by addition of $1 \mathrm{M}-\mathrm{HCl}$, was extracted twice with $0.2 \mathrm{vol}$. chloroform. After vigorous shaking and sedimentation, the chloroform extracts were combined, washed four times with water, filtered on anhydrous sodium sulphate, and evaporated under vacuum to dryness. The residue was dissolved in $10 \mathrm{ml}$ methanol and the soluble material fractionated in methanol on a column $(45 \times 2.5 \mathrm{~cm})$ of Sephadex LH-20. The different fractions were analysed by spotting $5 \mu \mathrm{l}$ of each on to a cellulose plate sprayed with an iron solution $\left(0 \cdot 1 \mathrm{M}-\mathrm{FeCl}_{3}\right)$ or with the phenolate spray reagent ( 1 vol. iron solution added to 1 vol. $0 \cdot 1 \mathrm{M}$-potassium ferricyanide) as described by Cox \& Graham (1979). The same procedure was used to purify pyochelin from $P$. aeruginosa ATCC 15692.

Physico-chemical analysis. UV-visible spectra of the purified compounds solubilized in methanol were obtained with a Pye-Unicam SP8-400 spectrophotometer. ${ }^{1} \mathrm{H}-\mathrm{NMR}$ analysis of cepabactin in $\mathrm{CD}_{3} \mathrm{OD}$ was done with a Bruker SY200 spectrometer. Thin-layer chromatography (TLC) on Polygram SIL G/UV 254 from Macherey and Nagel (Düren, FRG) was performed using eight different solvent systems: A, chloroform/methanol $(4: 1, v / v) ; B$, benzene/ethanol $(4: 1, \mathrm{v} / \mathrm{v}) ; \mathrm{C}$, benzene/ethyl acetate $(1: 9, \mathrm{v} / \mathrm{v}) ; \mathrm{D}$, benzene/acetone $(3: 1, \mathrm{v} / \mathrm{v}) ; \mathrm{E}$, chloroform/acetic acid $(90: 1, \mathrm{v} / \mathrm{v}) ; \mathrm{F}$, chloroform/ethanol $(4: 1, \mathrm{v} / \mathrm{v}) ; \mathrm{G}$, chloroform/acetic acid/ethanol $(90: 5: 2 \cdot 5$, by vol.); and H, 2-propanol $/ 25 \%$ ammonium hydroxide/water (100:10:10, by vol.). Systems A-D were those of Itoh et al. (1979), and systems E-H those of Cox \& Graham (1979).

\section{RESULTS}

\section{Growth and siderophore synthesis as a function of the iron concentration of the medium}

Cultures of $P$. cepacia in succinate medium reached the stationary phase after about $40 \mathrm{~h}$ of growth under the conditions used. Supplementation of the medium with iron $\left(\mathrm{FeCl}_{3}\right)$ increased both growth rate and cell yield (Fig. 1). Siderophore production was followed colorimetrically as described in Methods. The orange colour which developed immediately upon addition of an excess of iron to the culture was quantified by measuring, after centrifugation, the absorbance of the supernatant at $450 \mathrm{~nm}$. This absorbance increased progressively during exponential growth in succinate medium, and reached a maximal value when the culture entered the stationary phase. As shown in Fig. 2, which represents maximal growth yield and maximal siderophore production obtained in succinate media containing increasing concentrations of iron, the cell yield was proportional to the added iron concentration up to $4 \mu \mathrm{M}$ added iron. Production of siderophore was maximal with $2 \mu \mathrm{M}$-iron supplementation, decreasing markedly at higher iron concentrations. At $10 \mu \mathrm{M}$ added iron the siderophore was undetectable in the culture supernatant. The production of siderophore per unit of cells (expressed as the ratio $A_{450} / \mathrm{OD}_{650}$ ) followed a regular inverse relationship with the iron concentration of the growth medium until growth was no longer iron limited. This ratio was quite stable for iron-free succinate cultures incubated at different temperatures, within the range $25-37^{\circ} \mathrm{C}$, where the growth yield stayed constant. Above $37^{\circ} \mathrm{C}$, the ratio decreased drastically, and siderophore production was completely abolished at $41^{\circ} \mathrm{C}$, the growth temperature limit found for this bacterium (data not shown).

\section{Characterization of cepabactin and pyochelin}

The extraction of the chloroform-soluble material from 4 litres of culture supernatant of $P$. cepacia (succinate medium supplemented with $2 \mu \mathrm{M}$-iron, acidified to $\mathrm{pH} 3$ ) yielded $482 \mathrm{mg}$ of dry residue. When the methanol-soluble portion of this residue was applied to an LH-20 column 


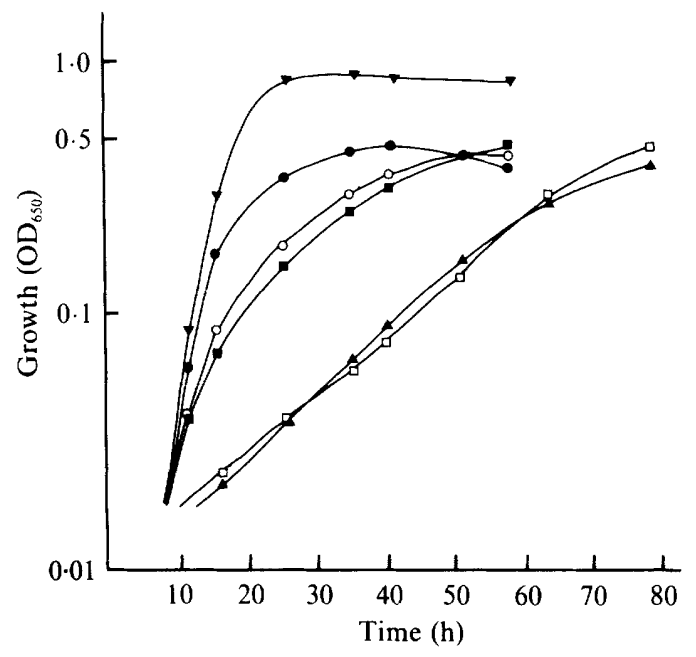

Fig. 1. Growth of $P$. cepacia ATCC 25416 in succinate medium $(\mathbb{a})$ or in the same medium supplemented with $20 \mu \mathrm{M}-\mathrm{FeCl}_{3}(\nabla)$ or $7 \mu \mathrm{M}$ cepabactin (O), $70 \mu \mathrm{M}$-cepabactin (O), 2.8 mM-EDDHA $(\square)$, or $20 \mu \mathrm{M}$-pyoverdine (isolated from P. fluorescens ATCC 13525) $(\boldsymbol{\Lambda})$. The results are means of triplicate experiments.

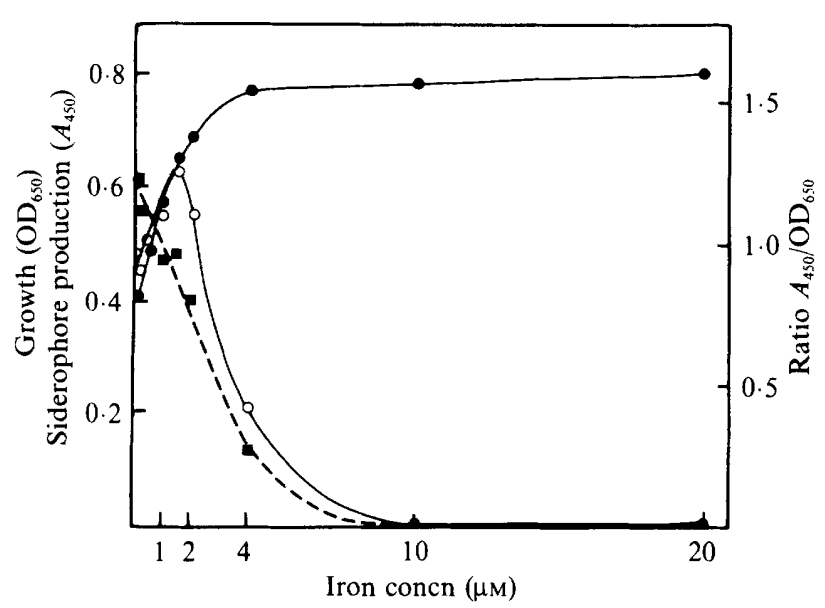

Fig. 2. Growth $\left(\mathrm{OD}_{650}, O\right)$ and siderophore production $\left(A_{450}, O\right)$ of $P$. cepacia as a function of the iron concentration of the culture medium. $\mathbf{b}$, Production of siderophore per unit of cells, expressed as the ratio $A_{450} / \mathrm{OD}_{650}$. Growth and siderophore production values are the means of triplicate experiments.

and eluted with methanol, an orange material eluted first, well separated from a yellow peak characterized by its weak fluorescence when illuminated with UV light at $365 \mathrm{~nm}$. Only the fractions constituting these two major peaks reacted positively with the $\mathrm{FeCl}_{3}$ and the phenolate spray reagents. They contained $182 \mathrm{mg}$ for the orange material (compound I) and $132 \mathrm{mg}$ for the yellow one (compound II). When the same procedure was applied to 5 litres of culture supernatant, acidified to $\mathrm{pH} 3$, of $P$. aeruginosa ATCC 15692, three separate peaks were obtained. The first peak was slightly purple, and contained $4 \mathrm{mg}$ of dry material; the two others, both yellow, contained 55 and $14 \mathrm{mg}$ respectively. Only the material collected as the major yellow peak gave a positive reaction with the two spray reagents and thus was further characterized; the two other peak fractions were discarded.

UV-visible spectra were obtained for methanol-solubilized samples of the three iron-reacting compounds isolated. Compound I from P. cepacia was characterized by a maximum absorption 
Table 1. TLC $R_{F}$ values of cepabactin and pyochelin

\begin{tabular}{|c|c|c|c|c|c|c|c|c|}
\hline \multirow[b]{3}{*}{ Compound } & \multicolumn{8}{|c|}{$R_{F}$ value } \\
\hline & \multicolumn{8}{|c|}{ Solvent system*: } \\
\hline & A & B & $\mathrm{C}$ & D & $\mathrm{E}$ & $\mathrm{F}$ & $\mathrm{G}$ & $\mathrm{H}$ \\
\hline $\begin{array}{l}\text { P. cepacia peak I } \\
\text { (cepabactin) }\end{array}$ & $\begin{array}{c}0.84 \\
(0.69) \dagger\end{array}$ & $\begin{array}{l}0.56 \\
(0.54) \dagger\end{array}$ & $\begin{array}{l}0.05 \\
(0.07) \dagger\end{array}$ & $\begin{array}{l}0.16 \\
(0 \cdot 22) \dagger\end{array}$ & $0 \cdot 19$ & 0.95 & $0 \cdot 51$ & $0 \cdot 17$ \\
\hline $\begin{array}{l}\text { P. cepacia peak II } \ddagger \\
\text { (pyochelin) }\end{array}$ & 0.73 & 0.47 & 0.19 & 0.22 & $0 \cdot 13$ & $0 \cdot 72$ & $0 \cdot 47$ & 0.46 \\
\hline $\begin{array}{l}P \text {. aeruginosa } \\
\text { pyochelin }\end{array}$ & 0.73 & 0.47 & $0 \cdot 18$ & $0 \cdot 21$ & $\begin{array}{c}0 \cdot 13 \\
(0 \cdot 19) \S\end{array}$ & $\begin{array}{c}0.71 \\
(0 \cdot 75) \S\end{array}$ & $\begin{array}{l}0.47 \\
(0.69) \S\end{array}$ & $\begin{array}{l}0.46 \\
(0 \cdot 60) \S\end{array}$ \\
\hline
\end{tabular}

at $330 \mathrm{~nm}$ and a weak absorption at $440 \mathrm{~nm}$ responsible for the typical orange colour of the concentrated solution. Compound II from $P$. cepacia, and the product isolated from $P$. aeruginosa, had UV-visible absorption spectra identical to those of pyochelin isolated from $P$. aeruginosa (Cox \& Graham, 1979) or from clinical isolates of $P$. cepacia (Sokol, 1986), with peaks at 210,250 and $310 \mathrm{~nm}$. Furthermore, both compounds had identical $R_{F}$ values when analysed on TLC plates, whereas $P$. cepacia compound I demonstrated specific $R_{F}$ values for each of the eight TLC-solvent systems used (Table 1). The chromatograms also revealed the homogeneity of compound I, which migrated as a single iron-reacting spot in each solvent system. In contrast, $P$. cepacia compound II and pyochelin both usually gave two or three iron-reacting spots, with one being predominant. This behaviour fitted well with the lability on TLC described by Cox \& Graham (1979) for pyochelin. Solvents A to D were those used by Itoh et al. (1979) to characterize the iron-chelating compound 1-hydroxy-5-methoxy-6-methyl-2(1H)-pyridinone produced by the soil isolate Pseudomonas $\mathrm{BN}-227$, whereas solvents $\mathrm{E}$ to $\mathrm{H}$ were those used by Cox \& Graham (1979) for the characterization of pyochelin from $P$. aeruginosa strains. The respective values found by these authors (indicated in parentheses in Table 1) roughly corresponded to compound I for the Pseudomonas BN-227 product, and to the yellow ironreacting compound of $P$. cepacia or $P$. aeruginosa for pyochelin, the few discrepancies probably being due to the differences in TLC supports, which can influence the mobility and the degree of lability of the tested compounds, especially pyochelin. For example, the chromatograms developed with solvents $\mathrm{G}$ and $\mathrm{H}$ revealed, on the Polygram SIL G/UV ${ }_{254}$ pre-coated plastic sheets used in this study, very minor spots for the $P$. cepacia compound II and the $P$. aeruginosa product, having $R_{F}$ values of respectively 0.73 and 0.57 , which corresponded more closely to the values $(0.69$ and 0.60$)$ obtained by Cox \& Graham (1979).

Since compound I of $P$. cepacia ATCC 25416 has never been previously described for welldefined $P$. cepacia strains, its possible identity as 1-hydroxy-5-methoxy-6-methyl- $2(1 \mathrm{H})$ pyridinone was further analysed by ${ }^{1} \mathrm{H}-\mathrm{NMR}$ spectroscopy. The characteristics of its spectrum when dissolved in $\mathrm{CD}_{3} \mathrm{OD}$ are given in Table 2, together with the attributed formula of the compound. The perfect correlation between these results and those of Itoh et al (1980) and Barker et al. (1979), together with the similarity shown by UV-visible spectrometry and TLC, led to the conclusion that $P$. cepacia compound I, which we term cepabactin, was 1-hydroxy-5methoxy-6-methyl-2 $(1 \mathrm{H})$-pyridinone, whereas $P$. cepacia compound II corresponded to pyochelin, already described as a potent siderophore for some clinical isolates of $P$. cepacia (Sokol, 1986).

\section{Effects of cepabactin and other ligands on growth of $P$. cepacia}

Supplementation of the succinate medium with cepabactin resulted in a growth stimulation which was proportional to the amount of ligand added. Stimulation was already effective with $7 \mu \mathrm{M}$-cepabactin (Fig. 1), and it increased linearly with increasing concentration of added 
Table 2. ${ }^{1} \mathrm{H}-\mathrm{NMR}$ spectrum of cepabactin in $\mathrm{CD}_{3} \mathrm{OD}$<smiles></smiles>

\begin{tabular}{lccccc} 
& $\mathrm{CH}_{3}$ & $\mathrm{OCH}_{3}$ & $\mathrm{H}_{a} \stackrel{x}{\longrightarrow}$ & $\mathrm{H}_{b}$ \\
$\delta$ (p.p.m.) & 2.44 & 3.81 & 6.59 & 7.45 \\
Multiplicity & $\mathrm{s}$ & $\mathrm{s}$ & $\mathrm{d}(7.5 \mathrm{~Hz})$ & $\mathrm{d}(7.9 \mathrm{~Hz})$ \\
\multicolumn{5}{c}{, Peaks not assigned. }
\end{tabular}

cepabactin, the maximal effect being observed at $70 \mu \mathrm{M}$-cepabactin, a concentration corresponding to the estimated limit of solubility of this compound in aqueous solution at $25^{\circ} \mathrm{C}$. Since the cepabactin was added as a methanolic solution it was checked that, at the concentrations used, the solvent had no effect on the bacterial growth (data not shown). Fig. 1 shows that the growth rate during the first hours of exponential growth was similar in succinate medium supplemented with $20 \mu \mathrm{M}-\mathrm{FeCl}_{3}$ or with $70 \mu \mathrm{M}$-cepabactin. Maximal cell yield, however, was doubled in the iron-supplemented medium compared to the unsupplemented and cepabactin-supplemented media, which gave about the same yield of biomass. Effects of several other iron chelators were also studied. Growth inhibition, resulting from a drastic lowering of growth rate together with an increased lag phase, was observed when succinate medium was supplemented with the synthetic iron chelator EDDHA (final concentration $2.8 \mathrm{mM}$ ) or with pyoverdine (final concentration $20 \mu \mathrm{M}$ ). Fig. 1 shows the results obtained with pyoverdine isolated from $P$. fluorescens ATCC 13525. Using pyoverdines from different bacterial strains, viz. $P$. fluorescens W (laboratory strain), $P$. fluorescens ATCC 17400 and $P$. aeruginosa ATCC 15692 (PAOl strain), which have slightly different chemical structures (Hohnadel \& Meyer, 1988), resulted in a similar inhibition effect. Identical results were obtained when $P$. cepacia was inoculated on to agar-solidified succinate medium supplemented with the various iron chelators. No growth appeared on plates supplemented with EDDHA (2.8 mM) even after one week of incubation, whereas $48 \mathrm{~h}$ at $25^{\circ} \mathrm{C}$ was sufficient to observe well-formed colonies on the unsupplemented medium. Pyoverdines tested by an impregnated $\left(7 \mu \mathrm{l}\right.$ of a $5 \mathrm{mg} \mathrm{ml}^{-1}$ aqueous solution) paper disc method (Hohnadel \& Meyer, 1988), gave a growth-inhibition zone around the disc that was still visible $2 \mathrm{~d}$ after the bacterial lawn had developed.

\section{Iron uptake mediated by cepabactin and other ligands}

When $P$. cepacia cells, harvested at the end of the exponential phase from succinate medium (iron-depleted cells), were incubated in non-proliferating conditions (succinate medium with the nitrogen source omitted) in the presence of ${ }^{59} \mathrm{Fe}$ chelated by cepabactin, uptake of labelled iron occurred rapidly, attaining 170 pmol ${ }^{59} \mathrm{Fe}$ per mg dry weight of cells after $30 \mathrm{~min}$ incubation (Fig. 3). The same experiment conducted at $0{ }^{\circ} \mathrm{C}$ instead of $25^{\circ} \mathrm{C}$ resulted in no iron uptake. Iron uptake was also completely inhibited when cells were washed and incubated at $25^{\circ} \mathrm{C}$ in a medium without succinate. Pre-incubation of the cells for $10 \mathrm{~min}$ in incubation medium plus $0.2 \mathrm{mM}-\mathrm{KCN}$ before the addition of the ${ }^{59} \mathrm{Fe}$ source resulted in a $73 \%$ inhibition of uptake, whereas $0.2 \mathrm{mM}$-DNP had only a slight effect (14\% inhibition). A $1 \mathrm{~mm}$ concentration of DNP had to be used in the incubation medium in order to observe a similar level of inhibition as that obtained with $0.2 \mathrm{~mm}-\mathrm{KCN}$ (Fig. 3). Cepabactin-mediated iron uptake by cells harvested from succinate medium supplemented with $20 \mu \mathrm{M}$-iron was inefficient. Incubation of iron-deficient cells with pyoverdines from different bacteria ( $P$. fluorescens $\mathrm{W}, P$. fluorescens ATCC 13525, $P$. fluorescens ATCC 17400, P. aeruginosa PAO1) or EDDHA instead of cepabactin as labelled iron chelator resulted in no uptake.

\section{DISCUSSION}

This study shows that cepabactin isolated from P. cepacia ATCC 25416 has all the characteristics of a siderophore: it is synthesized specifically by iron-deficient cells; it stimulates 


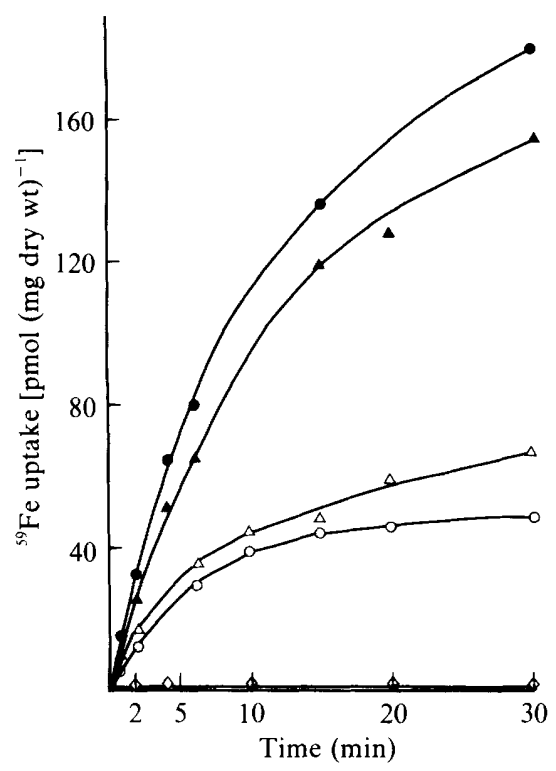

Fig. 3. Iron uptake in P. cepacia. Incorporation of ${ }^{59} \mathrm{Fe}$ chelated by cepabactin was measured with ironstarved cells resuspended in incubation medium at $25^{\circ} \mathrm{C}(\odot)$, at $0{ }^{\circ} \mathrm{C}(\diamond)$, or in incubation medium $\left(25^{\circ} \mathrm{C}\right)$ supplemented with $0.2 \mathrm{~mm}-\mathrm{DNP}(\Delta), 1 \mathrm{~mm}-\mathrm{DNP}(\triangle), 0.2 \mathrm{mM}-\mathrm{KCN}(O)$, or in incubation medium with the energy source (succinate) omitted $(\diamond)$. The symbol $(\diamond)$ also represents incorporation experiments performed in standard conditions (normal incubation medium, $25^{\circ} \mathrm{C},{ }^{59} \mathrm{Fe}$-cepabactin) but with cells previously grown in iron-rich medium, or with iron-starved cells but with cepabactin replaced by pyoverdine or EDDHA as iron chelators. Uptake assays were done at least in duplicate.

bacterial growth in an iron-poor medium and it specifically promotes iron uptake in irondeficient cells. In the presence of ferric ions, cepabactin formed an orange complex resulting from the presence in its structure of a hydroxamate group formed by the $N$-hydroxyl of a pyridine ring and the $N$-adjacent carbonyl group. Its full structure has been established by ${ }^{1} \mathrm{H}-\mathrm{NMR}$ to be identical to that of antibiotics G1549 and BN227, respectively synthesized by P. alcaligenes (Barker et al., 1979) and Pseudomonas BN227 (Itoh et al., 1979, 1980), and determined to be 1-hydroxy-6-methoxy-5-methyl-2(1H)-pyridinone. Moreover, the complexation properties of cepabactin, and its UV-visible spectrum and behaviour on TLC, were identical to those previously described for the two antibiotics. Thus, the two compounds G1549 and BN227 may be considered not only as antibiotics but also as siderophores of $P$. alcaligenes and Pseudomonas BN227. These two functions are not contradictory since it is well known that siderophores may have an antibiotic-like activity through their iron-chelating properties. Such behaviour has been shown in this study for the pyoverdines of various fluorescent pseudomonads, which considerably inhibited the growth of $P$. cepacia in liquid cultures and on agar plates. Since pyoverdines did not permit iron uptake by $P$. cepacia, their antibiotic-like effects are presumably the consequence of a higher affinity constant for iron compared to cepabactin. Another indication that compounds G1549 and BN227 possess their antibiotic activity thanks to their iron-complexation properties comes from a comparison of their MIC values and the MIC values of their iron chelates, determined by Barker et al. (1979) and Itoh et al. (1979) against a wide variety of micro-organisms. Usually the iron chelate had a much lower activity (higher MIC) and in some cases, no activity at all. Thus, cepabactin appears to be a siderophore not only for $P$. cepacia ATCC 25416, but also for other non-fluorescent pseudomonads.

By its structure, cepabactin can be considered as a cyclic hydroxamic acid, but also as a heterocyclic analogue of catechol. This compound thus occupies an interesting median position 
between the two siderophore families (hydroxamates and catecholates) usually recognized in the microbial world (Neilands, 1981). Synthetic hydroxy-pyridinones that have both cyclic hydroxamate and catechol-like structures have already been studied for their potent affinity and specificity for iron(III) (Scarrow, 1985): the overall formation constants for the iron(III)complexes of 1-hydroxy-2 $(1 \mathrm{H})$-pyridinone, 3-hydroxy-2 $(1 \mathrm{H})$-pyridinone and 3-hydroxy- $4(1 \mathrm{H})$ pyridinone were determined to be respectively $26 \cdot 9,29 \cdot 6$ and $35 \cdot 1$. The competition for iron between such synthetic compounds and transferrin has also been studied, and some derivatives [e.g. 1,2-dimethyl-3-hydroxy-4(1H)-pyridinone and 2-methyl-3-hydroxy-4(1H)-pyridinone] have been tested with success in in vivo experiments for iron removal (Kontoghiorghes, 1985). Whether cepabactin, which is a 1-hydroxy- $2(1 \mathrm{H})$-pyridinone derivative, the compound among the series with the lowest affinity for iron(III) (26.9), competes successfully for iron chelated by transferrin remains to be determined. In conclusion, since pyochelin has already been shown by Sokol (1986) to facilitate iron translocation in P. cepacia strains, it appears that this bacterium possess at least two different siderophore-mediated iron-uptake systems.

We gratefully acknowledge M. W. Hosseini for ${ }^{1} \mathrm{H}-\mathrm{NMR}$ spectra and helpful discussions, and G. Seyer for valuable technical assistance.

\section{REFERENCES}

Ankenbauer, R., Sriyosachati, S. \& Cox, C. D. (1985). Effects of siderophores on the growth of Pseudomonas aeruginosa in human serum and transferrin. Infection and Immunity 49, 132-140.

Barker, W. R., Callaghan, C., Hill, L., Nobel, D., ACred, P., Harper, P. B., Sowa, M. A. \& Fletton, R. A. (1979). G1549, a new cyclic hydroxamic acid antibiotic, isolated from culture broth of Pseudomonas alcaligenes. Journal of Antibiotics 32, 10961103.

Bickel, H., Bosshardt, R., Gaumann, E., Reusser, P., Vischer, E., Voser, W., WetTStein, A. \& ZÄHNER, H. (1960). Stoffwechsel Produkte von Actinomyceten. Über die Isolierung und Characterisierung der Ferrioxamine A-F, neuer Wuchstoffe der Sideramin Gruppe. Helvetica chimica acta 43, 2118 2128.

Bukovits, G. J., Mohr, N., Budzikiewicz, H., Korth, H. \& PUlverer, G. (1982). 2-phenyl thiazole derivatives from Pseudomonas cepacia. Zeitschrift für Naturforschung 37b, 877-880.

BURKHOLDER, W. H. (1950). Sour skin, a bacterial rot of onion bulbs. Phytopathology 40, 115-117.

Burnham, B. F. \& Neilands, J. B. (1961). Studies on the metabolic function of the ferrichrome compounds. Journal of Biological Chemistry 236, 554559.

CODY, Y. S. \& GRoss, D. C. (1987). Characterization of pyoverdin $_{\mathrm{pss}}$, the fluorescent siderophore produced by Pseudomonas syringae pv. syringae. Applied and Environmental Microbiology 53, 928-934.

Cox, C. D. \& GraHAM, R. (1979). Isolation of an ironbinding compound from Pseudomonas aeruginosa. Journal of Bacteriology 137, 357-364.

HOHNADEL, D. \& MEYER, J. M. (1988). Specificity of pyoverdine-mediated iron uptake among fluorescent Pseudomonas strains. Journal of Bacteriology 170, 4865-4873.

Holmes, B. (1986). The identification of Pseudomonas cepacia and its occurrence in clinical material. Journal of Applied Bacteriology 61, 299-314.
Itoh, J., MiYadoh, S., Takahasi, S., Amano, S., Ezaki, N. \& Yamada, Y. (1979). Studies on antibiotics $\mathrm{BN}-227$ and $\mathrm{BN}-227-\mathrm{F}$, new antibiotics. I. Taxonomy, isolation and characterization. Journal of Antibiotics 32, 1089-1095.

Itoh, J., Amano, S., Ogawa, Y., Kodama, Y., Ezaki, N. \& Yamada, Y. (1980). Studies on antibiotics BN227 and $\mathrm{BN}-227-\mathrm{F}$, new antibiotics. II. Chemical structure of antibiotics $\mathrm{BN}-227$ and $\mathrm{BN}-227-\mathrm{F}$. Journal of Antibiotics 33, 377-382.

Keller-Schierlein, W. \& Prelog, V. (1961). Stoffwechsel Produkte von Actinomyceten. Über das Ferrioxamin E; ein Beitrag zur Konstitution de Nocardamins. Helvetica chimica acta 44, 1981-1985.

KonTOGHIORGHES, G. J. (1985). New orally active iron chelators. Lancet 1, 817.

LEONG, J. (1986). Siderophores: their biochemistry and possible role in the biocontrol of plant pathogens. Annual Review of Phytopathology 24, 187-209.

LIU, P. V. \& ShokranI, F. (1978). Biological activities of pyochelins: iron-chelating agents of Pseudomonas aeruginosa. Infection and Immunity 22, 878-890.

Meyer, J. M. \& Abdallah, M. A. (1978). The fluorescent pigment of Pseudomonas fiuorescens: biosynthesis, purification and physico-chemical properties. Journal of General Microbiology 107, 319328.

Meyer, J. M. \& Abdallah, M. A. (1980). The siderochromes of non-fluorescent pseudomonads: production of nocardamine by Pseudomonas stutzeri. Journal of General Microbiology 118, 125-129.

Meyer, J. M. \& Hornsperger, J. M. (1978). Role of pyoverdine $_{\mathrm{p}}$, the iron binding fluorescent pigment of Pseudomonas fluorescens, in iron transport. Journal of General Microbiology 107, 329-331.

Meyer, J. M., Halle, F., Hohnadel, D., LemanceaU, P. \& Ratefiarivelo, H. (1987). Siderophores of Pseudomonas - biological properties. In Iron Transport in Microbes, Plants and Animals, pp. 188-205. Edited by G. Winkelmann, D. van der Helm \& J. B. Neilands. Weinheim: VCH Verlagsgesellschaft. 
MülleR, G. \& Raymond, K. N. (1984). Specificity and mechanism of ferrioxamine-mediated iron transport in Streptomyces pilosus. Journal of Bacteriology 160, 304-312.

MÜLLER, A. \& Z̈̈HNER, H. (1968). Stoffwechselprodukte von Mikroorganismen. Ferrioxamine aus Eubacteriales. Archiv für Mikrobiologie 62, 257-263.

NeIlands, J. B. (1981). Microbial iron compounds. Annual Review of Biochemistry 50, 715-731.

Palleroni, N. J., Kunisawa, R., Contopoulou, R. \& DoudorofF, M. (1973). Nucleic acid homologies in the genus Pseudomonas. International Journal of Systematic Bacteriology 23, 333-339.

PreloG, V. (1963). Iron containing antibiotics and microbic growth factors. Pure and Applied Chemistry 6, 327-338.

SCARROW, R. C. (1985). Hydroxypyridinones: ligands with high affinity and specificity for iron(III). $\mathrm{PhD}$ dissertation, University of California, Berkeley.

Sokol, P. A. (1984). Production of the ferripyochelin outer membrane receptor by Pseudomonas species. FEMS Microbiology Letters 23, 313-317.

Sokol, P. A. (1986). Production and utilization of pyochelin by clinical isolates of Pseudomonas cepacia. Journal of Clinical Microbiology 23, 560-562.

SoKoL, P. A. \& Woods, D. E. (1983). Demonstration of an iron-siderophore-binding protein in the outer membrane of Pseudomonas aeruginosa. Infection and Immunity 40, 665-669.

Stanier, R. Y., Palleroni, N. J. \& Doudoroff, M. (1966). The aerobic pseudomonads: a taxonomic study. Journal of General Microbiology 43, 159-271.

Stoll, A., Brack, A. \& RenZ, J. (1951). Nocardamin, ein neues Antibioticum aus einer Nocardia-Art. Schweizerische Zeitschrift für allgemeine Pathologie und Bakteriologie 14, 225-233.

Teintze, M., Hossain, M. B., Barnes, C. L., Leong, J. \& VAN DER HELM, D. (1981). Structure of ferric pseudobactin, a siderophore from a plant growth promoting Pseudomonas. Biochemistry 20, 64466457.

De Weger, L. A., van Arendonk, J. J. C. M., Recourt, K., VAN der Hofstad, G. A. J. M., WEISBEEK, P. J. \& LUGTENBERG, B. (1988). Siderophore-mediated uptake of $\mathrm{Fe}^{3+}$ by the plant growthstimulating Pseudomonas putida strain WCS358 and by other rhizosphere microorganisms. Journal of Bacteriology 170, 4693-4698.

ZäHNER, H., BACHMAN, E., HÜTTER, R. \& Nuesch, J. (1962). Sideramine, eisenhaltige Wachstumfaktoren aus Mikroorganismen. Pathologia et microbiologia 25, 708-736. 